\title{
Survie et maintien de la virulence de Salmonella Typhimurium VNC exposée simultanément à trois facteurs stressants expérimentaux
}

\author{
Bernard BALEUX ${ }^{a}$, Audrey CARO ${ }^{a}$, Jean LESNE $^{\text {b }}$, Patrice GOT ${ }^{\text {a }}$, Sylvie BINARD ${ }^{\text {b }}$, Bruno DELPEUCH ${ }^{\text {b }}$ \\ ${ }^{a}$ Laboratoire d'hydrobiologie et UMR CNRS, UM II 5556, université Montpellier-II. 34095 Montpellier cedex 5, France \\ ${ }^{\mathrm{b}}$ Laboratoire études et recherche en environnement et santé, École nationale de la Santé publique. 35043 Rennes cedex, \\ France
}

(Révisé le 24 juin 1998, accepté le 2 juillet 1998)

\begin{abstract}
Survival and virulence changes in the VNC state of Salmonella Typhimurium in relation to simultaneous UV radiation, salinity and nutrient deprivation exposure. The release of enteric pathogenic bacteria in aquatic environments poses the problem of the fate of these bacteria under the effects of environmental factors (solar radiation, salt concentration, temperature, nutrient level, $\mathrm{pH}$, competition). Frequently, these bacterial cells, potentially pathogenic, enter into a non-culturable state on routine bacteriological plating media. However, the use of direct detection methods (DAPI stained cells) allows the visualization of these Viable but Non Culturable cells (VNC). But, beyond the characterization of the viability of the cells (electron transport activity, metabolic activity. membrane integrity, structure and/or quantity of DNA), what happens with the virulence of these cells? This problem was experimentally investigated according to the bacterial model Salmonella Typhimurium. The virulence of this strain, which is the agent of the murine typhoid, was evaluated on a mouse model. Experimentally, the effects of some environmental factors on the survival and on the maintenance of virulence of Salmonella Typhimurium were measured in microcosms exposed to UV radiation (four germicidal lamps $8 \mathrm{~mW} \mathrm{~s}^{-1} \mathrm{~cm}^{-2}$, wave length: $254 \mathrm{~nm}$ ), salt concentration (Sea Salt Sigma, 37), nutrient starvation. The microcosms were simultaneously submitted to these three factors, with variable exposure times. For each of those times, the viability of the nonculturable cells (which became nonculturable because of the exposure to the three factors) was measured through different physiological states notable in the cells, after using different fluorescent dyes. The stained cells were observed by epifluorescence microscopy and analysed by image cytometry. So, the cellular populations are characterised by enumeration of respiring bacteria (CTC, [39]), metabolising bacteria (YEK, [22] modified), bacteria owning an undamaged cytoplasmic membrane (LD, Live/Dead BacLight Viability Kit. Molecular Probes Inc.); we also determined the quantity and/or structure of DNA of the cells (fluorescence level of DAPI stained cells).

After exposure to the three factors for one hour $\left(13.56 \mathrm{~J} \mathrm{~cm}^{-2}\right)$, while the plate count cell density rapidly decreased from $\# 10^{7}$ cells $\mathrm{mL}^{-1}$ to $<0.1$ cell $\mathrm{mL}^{-1}$, physiological states of these viable but non-culturable cells are similar to those of nonexposed cells. On the other hand, after exposure for three hours, only $10 \%$ of the cells deposit a CTC formazan-crystal and $20 \%$ are substrate responsive cells (enlarged cells in presence of Yeast Extract and Cephalexin: YEC). Half of the cellular population presents an undamaged cytoplasmic membrane and the level of fluorecence of DAPI stained cells is close to $85 \%$, which shows that the DNA of these cells is weakly damaged. After exposure to the three experimental factors for 24 hours $\left(315 \mathrm{~J} \mathrm{~cm}^{-2}\right)$, weak replies to the physiological tests used in this study to characterize the viability of the non-culturable cellular population are observed (CTC: $4 \%$; YEC: $2 \% ;$ LD: $11.8 \%$ ) while the fluorescence level of DAPI stained cells remains firm at $80 \%$. At the same time, the virulence expression of VNC cells of Salmonella Typhimurium, evaluated by intraperitoneal injection to the mouse (route which excludes uncontrolled parameters, unlike the per os route) does not seem to be correlated with the cellular viability such as it has been evaluated in this study. A 30 min exposure $\left(6.73 \mathrm{~J} \mathrm{~cm}^{-2}\right)$ to the three environmental factors, leading to the non-culturability of almost the entire exposed cell population $\left(0.08\right.$ culturable cell $\left.\mathrm{mL}^{-1}\right)$ whereas the level of viability of those culturable cells is closed to the one of non-
\end{abstract}


exposed cells. The injection of 1000 of those cells $(<0.001$ culturable cells in $100 \mu \mathrm{L}$ inoculated) into the mouse (a group of ten mice) does not cause any mortality four weeks post-inoculation, whereas the injection of the same dose of nonexposed cells leads to the death of all mice in the group one week post-inoculation. According to our preliminary experiments on Salmonella Typhimurium, the loss of the state of culturability and the loss of virulence towards mice by intraperitoneal route, because of the exposure to associated effects of UV irradiation (254 nm), salinity (37) and nutrient starvation, seem to be concomitant. (1) Elsevier, Paris

\section{Salmonella / survival / virulence / VNC / stress}

Résumé - Le rejet de bactéries entériques pathogènes dans l'environnement aquatique pose le problème du devenir de ces microorganismes sous les effets des facteurs environnementaux (rayonnement solaire, salinité. température, oligotrophie, $\mathrm{pH}$, compétition). L'évolution fréquente de ces cellules bactériennes potentiellement pathogènes vers un état non cultivable implique l'absence de détection de celles-ci par des méthodes traditionnelles. Cependant, l'utilisation des méthodes de détection directes permet de visualiser ces cellules non cultivables. Au stade suivant, il est nécessaire de caractériser le niveau de viabilité de ces cellules non cultivables. Mais au-delà de la caractérisation de l'état viable de la cellule (activité des chaînes respiratoires, activité métabolique, intégrité membranaire, structure et/ou quantité d'ADN) se pose parallèlement le problème du maintien du pouvoir pathogène de ces bactéries. Cette problématique a été abordée expérimentalement sur le modèle bactérien Salmonella Typhimurium. Pour évaluer le pouvoir pathogène de cette souche, il est fait appel au modèle souris, Salmonella Typhimurium étant l'agent de la typhoïde murine. Pour évaluer les effets de certains facteurs environnementaux sur cette souche pathogène, il a été fait appel sur le plan expérimental en microcosmes au rayonnement UV (quatre lampes germicides $8 \mathrm{~mW} \mathrm{~s}^{-1} \mathrm{~cm}^{-2}$ à $254 \mathrm{~nm}$ ), à la salinité (SeaSalt Sigma, 37) et à l'oligotrophie. Ces trois facteurs sont appliqués simultanément avec des temps d'exposition variables. Pour chaque temps d'exposition, le niveau de viabilité des cellules qui ont perdu le pouvoir de cultiver sous les effets de ces facteurs est mesuré à travers différents états physiologiques, au moyen de marqueurs fluorochromiques. La réaction marqueur-cellule est observée par microscopie en épifluorescence et analysée par cytométtie en images. Ainsi, les populations cellulaires sont caractérisées sur le plan de la respiration (CTC, [39]), de la capacité à métaboliser un nutriment (YEC, [22] modifié), de l'intégrité membranairc (LD. Kit Live/Dead Baclight- Molecular Probes Inc) et de la structure et/ou quantité d'ADN [36]. Alors que la disparition du pouvoir de cultiver de toutes les cellules exposées (zéro cultivabilité) se réalise en $I \mathrm{~h}\left(13,56 \mathrm{~J} \mathrm{~cm}^{-2}\right)$, les états physiologiques que présentent ces cellules non cultivables sont proches de ceux présentés par les cellules d'une culture non exposée. Par contre, après $3 \mathrm{~h}$ d'exposition, 10 \% seulement des cellules présentent une activité de leur chaîne respiratoire (CTC) et $20 \%$ présentent une activité métabolique (extrait de levure en présence de céphalexine). La moitié des cellules présente une atteinte de la membrane cytoplasmique et le niveau de fluorescence de la population cellulaire est voisin de $85 \%$ montrant par là que ces cellules ne présentent à ce stade qu'une faible atteinte de l'ADN. Après $24 \mathrm{~h}$ d'exposition $\left(315 \mathrm{~J} \mathrm{~cm}^{-2}\right)$ à ces trois facteurs stressants expérimentaux, les tests physiologiques appliqués dans cette étude sur les cellules non cultivables sont quasiment négatifs alors que le niveau de fluorescence de leur ADN marqué se mainticnt à $80 \%$. En parallèle, le suivi du pouvoir pathogène de ces cellules de Salmonella Typhimurium, évalué par injection intrapéritonéale à la souris (voie permettant de s'affranchir de paramètres non contrôlés comme la voie per os), ne paraît pas évolucr en fonction de la disparition de la viabilité cellulaire mesurée par les tests utilisés dans cette étude. Une exposition de $30 \mathrm{~min}\left(6,73 \mathrm{~J} \mathrm{~cm}^{-2}\right)$ aux trois facteurs stressants expérimentaux fait disparâtre le pouvoir de cultiver de la quasi-totalité des cellules exposées $\left(0,08\right.$ cellules cultivables $\left.\mathrm{mL}^{-1}\right)$ alors que ces cellules présentent un état de viabilité proche de celui d'une souche non exposée. L'injection de 1000 de ces cellules $(<0,001$ cellule cultivable dans $100 \mu \mathrm{L}$ injecté) à la souris (un lot de dix souris) n'entraîne aucun état pathologique ni mortalité sur quatre semaines d'observations alors que l'injection du même nombre de cellules non exposées aux trois facteurs stressants entrâne la mort du lot de souris en une semaine.

D'après ces quelques expérimentations prélininaires sur Salmonella Typhimurium, la perte du pouvoir de cultiver paraît s'accompagner de la perte de la virulence testée chez la souris par voie intrapéritonéale après exposition aux effets associés de l'irradiation UV, de la salinité 37 et de l'oligotrophie. (.) Elsevier, Paris

Salmonella / survie / virulence / VNC / stress 


\section{INTRODUCTION}

En environnements aquatiques expérimentaux (microcosmes) il a été possible de mettre en évidence des cellules d'entérobactéries non détectables par les méthodes culturales indirectes (MPN, heterotrophic plate count [18]) en faisant appel à de nouvelles méthodes directes telles que les marquages cellulaires fluorescents (A.O, DAPI) $[5,15,43]$, l'immunolluorescence [12], la PCR $[4]$.

L'exposition aux facteurs environnementaux tels que la carence nutritive, la température, la salinité [27]. la lumière visible $[3,16]$ de cellules bactériennes issues d'un tube digestif, conduit plus ou moins rapidement à la perte du pouvoir de cultiver de ces cellules. Bien que non cultivables, une fraction importante des cellules bactériennes conserve une certaine activité physiologique, en particulier la capacité de métaboliser un nutriment, mise en évidence par le Direct Viable Count (DVC) $[22,42]$ : elles sont décrites comme viables non cultivables (VNC). Cet état de dormance cellulaire qui peut s'accompagner de changement de taille ou de forme des cellules $[40,41]$ conditionne leur survie à long terme dans l'environnement aquatique.

L'état viable mais non cultivable a été étudié sur des bactéries pathogènes par différents auteurs: Aeromonas salmonicida [1]. Campylobacter jejuni [21, 40], Escherichia coli enterotoxinogène [45, 46], Salmonella enteritidis [43], Salmonella paratyphi, Staphylococcus [9]. Vibrio cholerae [7, 48], Vibrio vulnificus [31], Yersinia enterocolitica [44].

Plus récemment, certains auteurs ont étudié la pathogénie des cellules bactériennes pathogènes viables mais non cultivables: Shigella dysenteriae Type 1 [37, 38], Vibrio cholerae 01 [8], Vibrio vulnificus $[26,32,33]$.

La pathogénie potentielle des cellules bactériennes pathogènes viables mais non cultivables paraît mériter une attention toute particulière à cause de son intérêt en santé publique.

Le but de cette étude dont les résultats préliminaires sont présentés dans cette publication est d'évaluer les effets de l'application simultanée de trois facteurs de stress (rayonnement UV, salinité, oligotrophie) :

- sur la perte du pouvoir de cultiver des cellules d'une souche de Salmonella Typhimurium,

- sur la caractérisation par cytométrie en images des états physiologiques que présentent ces cellules viables mais non cultivables,
- sur la conservation ou non du pouvoir pathogène des cellules VNC de cette souche bactérienne pathogène testée sur modèle animal (injection intrapéritonéale à la souris).

\section{MATÉRIELS ET MÉTHODES}

\subsection{Souche bactérienne}

Salmonella Typhimurium C52, dérivée de la souche sauvage $C 5$, utilisée dans cette étude a été gracieusement mise à notre disposition par M. le $\mathrm{P}^{r}$ Popoff (Institut Pasteur. Paris). Elle possède les caractères métaboliques typiques du genre Salmonella et ses caractères antigéniques sont les suivants: OMA+, O:4.5+, HMA+, H:i+, H:1,2+. De plus, Salmonella Typhimurium C52 porte un plasmide cryptique de $90 \mathrm{~kb}$, associé au pouvoir pathogène de cette souche. Cette dernière engendre chez la souris la typhoïde murine (proche de la typhoïde humaine) entraînant la mort par septicémie.

Une culture de Salmonella Typhimurium C52 en bouillon Trypcase Soja $\left(37^{\circ} \mathrm{C}, 18 \mathrm{~h}\right.$ ) (bioMérieux), centrifugée (3000 rpm/10 min) et lavée trois fois en tampon TRISHCl pH 7, I (TRIS, Sigma), constitue l'inoculum bactérien utilisé pour ensemencer les microcosmes.

\subsection{Protocole expérimental}

Les microcosmes sont constitués de cristallisoirs en pyrex (diamètre de $30 \mathrm{~cm}$ ), contenant chacun un litre d'eau de mer artificielle ( $40 \mathrm{~g}$ de Sea Salt-Sigma par litre d'eau distillée, soit 37 de salinité), stérilisée par filtration sur membrane Nuclépore ( $0,2 \mu \mathrm{m}$ de porosité). La hauteur d'eau dans les microcosmes est environ de $3 \mathrm{~cm}$. Chaque microcosme, soumis à une agitation magnétique constante après ajout de l'inoculum bactérien, titre \# $10^{7}$ cellules $\mathrm{mL}^{-1}$.

Le dispositif expérimental de l'exposition des microcosmes au rayonnement UV (254 nm) consiste en quatre lampes germicides $\left(8 \mathrm{~mW} \mathrm{~s}^{-1} \mathrm{~cm}^{-2}\right)$ montées en série et disposées à $30 \mathrm{~cm}$ au-dessus de la surface liquide des microcosmes

Dans un premier temps, un microcosme est utilisé préalablement pour mesurer la cinétique des différents états physiologiques cellulaires et des concentrations en cellules totales et cultivables de la population de Salmonella 
Typhimurium C52 soumise simultanément aux stress rayonnement $\mathrm{UV}$, salinité, oligotrophie.

Dans un deuxième temps, compte tenu de cette cinétique, trois temps d'exposition à ces facteurs stressants $(30 \mathrm{~min}$, 1 h et 24 h en double) ont été retenus. Ceci vise à obtenir des populations bactériennes dans lesquelles les cellules se présentent dans des états physiologiques différents. Après chaque temps d'exposition, $200 \mathrm{~mL}$ de chaque microcosme sont centrifugés $(3000 \mathrm{rpm} / 10 \mathrm{~min})$ et lavés (tampon Tris-HCl pH 7,1) trois fois. À partir de ce culot, sont réalisées les suspensions bactériennes stressées (tampon Tris) qui seront testées vis à vis du pouvoir pathogène sur le modèle animal (souris).

\subsection{Dénombrement et mesure des états physiologi- ques des cellules bactériennes totales}

Le dénombrement des cellules bactériennes, cultivables et totales, ainsi que la mesure de leurs états physiologiques sont réalisés au moment de l'introduction de l'inoculum dans les microcosmes, ainsi que dans les microcosmes avant exposition aux facteurs stressants. À la fin de l'exposition à ces lacteurs et avant l'injection aux souris, les cellules éventuellement cultivables, et en parallèle les cellules VNC (cultivabilité niveau « zéro »), sont également dénombrées.

Les cellules de Salmonella Typhimurium C52 cultivables sont dénombrées soit par étalement sur gélose nutritive (GN, bioMérieux), soit par filtration (Membrane Gelman Science, GN6 Grid. de porosité $0,45 \mu \mathrm{m}$ ) de la totalité de la suspension restante du microcosme; la membrane filtrante est incubée sur gélose nutritive $\left(37^{\circ} \mathrm{C} / 24 \mathrm{~h}\right)$.

Le dénombrement et la mesure des états physiologiques des cellules totales marquées par les fluorochromes se fait de façon standardisée au moyen d'un microscope à épifluorescence couplé à un analyseur d'images $\{15\}$. Ceci permet une approche cellulaire objective d'une population bactérienne basée sur une visualisation cellulaire individuelle [2].

Le dénombrement des cellules totales est effectué par marquage au fluorochrome DAPI (4'.6-Diamidino-2-phényl-indole, Sigma) [36]. L'observation microscopique est réalisée selon le protocole décrit par [2].

La mesure des états physiologiques des cellules bactériennes totales comprend la mesure des activités respiratoire et métabolique, de l'intensité de fluorescence de l'ADN et de l'intégrité de la membrane cytoplasmique.
L'activilé respiratoire de chaque cellule dénombrée est appréciée par la réduction du CTC (5-cyano-2,3-ditolyl tetrazolium chloride) [39]. L'activité métabolique des cellules est mesurée par une variante de la méthode décrite par [22]. L'antibiotique utilisé dans cette variante est la céphalexine [2].

L'intensité de la fluorescence réémise des cellules marquées au DAPI dépend de la fïxation du fluorochrome sur les bases A-T de l'ADN et peut refléter son état structural et/ou son abondance. Ce niveau de fluorescence est apprécié sur une échelle de 256 niveaux de gris par l'analyseur d'images [2].

L'évaluation de l'intégrité de la membrane cytoplasmique est basée sur la pénétration passive intracellulaire de deux fluorochromes (Kit Live/Dead Baclight-Molecular Probes Inc). Selon le protocole décrit par Molecular Probes, après excitation des cellules marquées, la fluorescence rouge des cellules est le signe d'une altération membranaire alors que la fluorescence verte met en évidence l'intégrité de la membrane cytoplasmique.

\subsection{Infection animale}

La lignée pure de souris $\mathrm{C} 57 \mathrm{Bl} / 6 \mathrm{~J} / \mathrm{Rj}$ a été retenue pour sa très grande sensibilité à Salmonella Typhimurium. Ces souris sont consanguines hétéroxéniques (specific-pathogen free); elles sont fournies par le CERJ, 53940 Le Genest-sur-Isle, France.

Le pouvoir pathogène des cellules de Salmonella Typhimurium soumises aux trois facteurs environnementaux sera évalué par la mesure du pourcentage de mortalité à la dose létale 100 (DL100) après injection intrapéritonéale chez les souris $\mathrm{C} 57 \mathrm{~B} 1 / 6 \mathrm{~J} / \mathrm{Rj}$.

Le choix de l'injection intrapéritonéale s'est appuyé sur des essais préalables ayant permis de conclure à une meilleure sensibilité (DL $100<10^{2}$ cellules), reproductibilité et rapidité de réponse de cette voie par rapport à la voie d'injection per os (DL50 évalućc à $10^{4}$ cellules et DL100) estimée par extrapolation supérieure à $6 \times 10^{6}$ cellules).

L'inoculation intrapéritonéale de $100 \mu \mathrm{L}$ d'une suspension de cellules bactériennes stressées (\# $10^{3}$ cellules) est effectuée sur un lot de dix souris âgées de six semaines. Parallèlement, une même quantité de cellules provenant d'une suspension de Salmonella Typhimurium C52 non soumise aux trois facteurs stressants (témoin pathogène), est injectée à un autre lot de six souris. Les souris sont isolées après inoculation. 
La mortalité des souris inoculées est suivie quotidiennement pendant quatre semaines. Les souris mortes sont autopsiées. Le foie et la rate sont mis en culture. Les colonies développées sont identifiées par séroagglutination sur lame. Au bout de quatre semaines, les souris survivantes sont sacrifiées (asphyxie au $\mathrm{CO}_{2}$ ) et leurs broyats foie-rate sont mis en culture.

\section{RÉSULTATS}

Cette étude vise, à travers quelques essais, à tester sur un modèle animal (souris), le pouvoir pathogène des cellules de Salmonella Typhimurium C52 viables non cultivables après exposition, plus ou moins prolongée, au rayonnement UV dans un environnement salin (37) et oligotrophe. La variation du temps d'exposition permet d'obtenir des populations cellulaires VNC présentant des états physiologiques différents.

La cinétique de l'évolution des dénombrements des cellules cultivables et des cellules totales d'une part, et des mesures des états physiologiques des cellules totales en fonction du temps d'exposition aux facteurs stressants d'autre part, ( 1 h, 2 h, 3 h, et 24 h) est représentée sur la figure 1.

Dès $1 \mathrm{~h}$ d'exposition aux trois facteurs stressants, le dénombrement des cellules cultivables de Salmonella Typhimurium $\mathrm{C} 52$ devient négligeable $(0,03$ cellule $\mathrm{mL}^{-1}$ ).

Quelque soit le temps d'exposition aux facteurs stressants, l'abondance des cellules totales, évaluée par la méthode directe DAPI, reste constante.

Avant l'exposition aux facteurs stressants (T0), les mesures des activités respiratoire, métabolique et de l'intégrité membranaire sont inférieures à $100 \%$ (respectivement $66,7,67$ et $91 \%$ ). Ceci est en fait lié aux trois centrifugations et lavages effectués lors de la préparation de l'inoculum bactérien utilisé pour ensemencer les microcosmes. Après exposition, les différents états physiologiques considérés évoluent en fonction du temps d'exposition et d'une façon hiérarchisée. En 1 h d'exposition, les valeurs

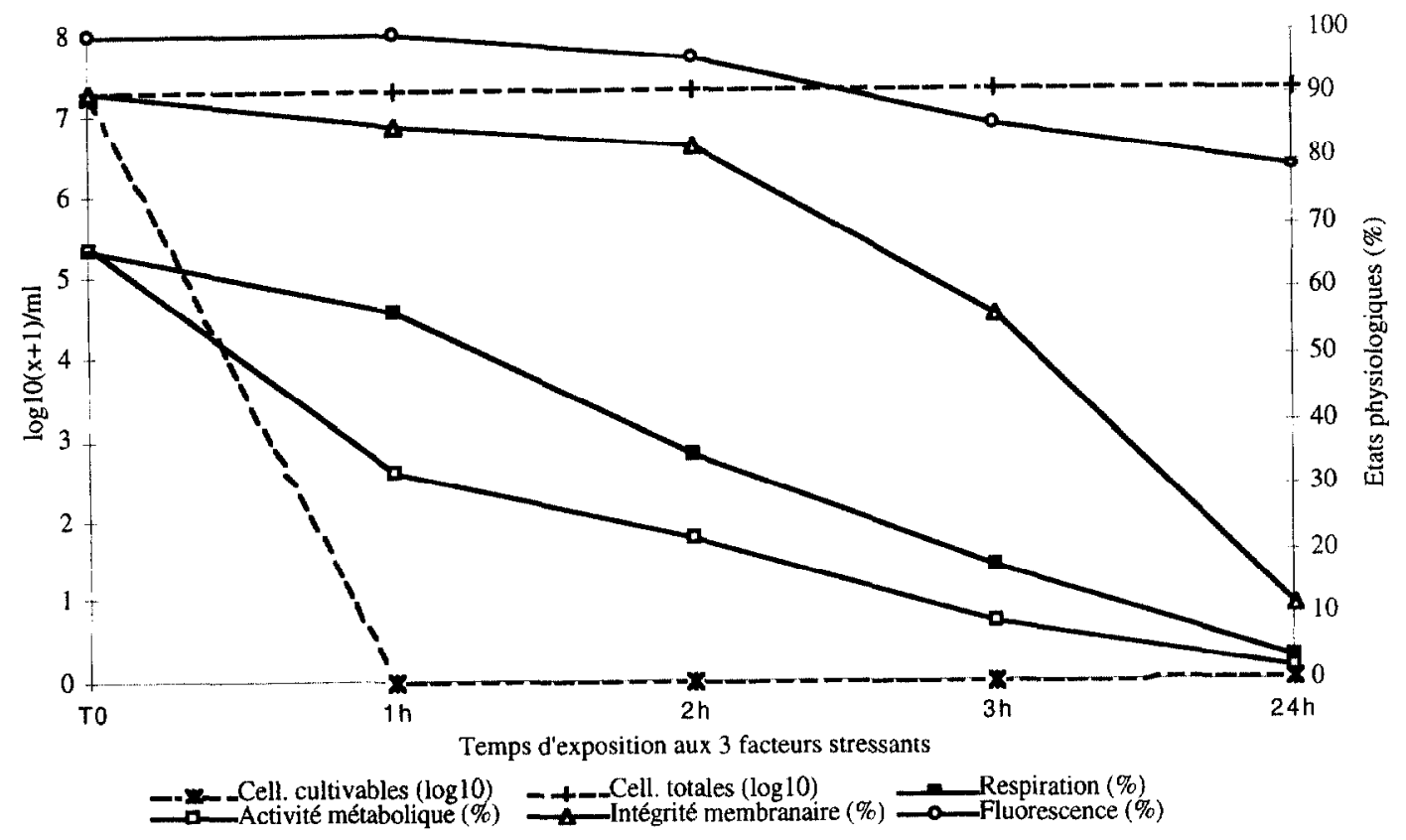

Figure 1. Évolution temporelle des abondances $(\log 10)$ des cellules totales et cultivables ainsi que des pourcentages des états physiologiques cellulaires d'une population de Salmonella Typhimurium C52 exposée simultanément aux trois facteurs stressants (oligotrophie, salinité et rayonnement UV). T0 : cellules de Salmonella Typhimurium C52 non exposées aux facteurs stresssants.

Figure 1. Culturable and total counts $(\log 10)$ and physiological states $(\%)$ of Salmonella Typhimurium $\mathrm{C} 52$ cellular population over time under the effects of simultaneous exposure to the three experimental factors (UV radiation, salinity, nutrient starvation). 
des mesures des activités respiratoire et métabolique diminuent et passent respectivement à 57 et à $32 \%$. Pendant le même temps d'exposition, la valeur de l'intégrité membranaire est faiblement affectée, de même que celle de la fluorescence qui demeure égale à $100 \%$.

Lors d'une exposition d'une durée de 2 h, l'altération des activités respiratoire et métabolique s'accentue (35 et $22 \%$ ) et l'intégrité membranaire, bien que présentant une valeur élevée ( $>80 \%$ ), diminue progressivement. L'altération du niveau de réémission de la fluorescence ne débute qu'à partir d'une exposition de $2 \mathrm{~h}$ aux facteurs stressants.

Après $3 \mathrm{~h}$ d'exposition, l'altération des activités respiratoire et métabolique s'accentue (18 et $9 \%$ ) mais c'est surtout l'atteinte de l'intégrité membranaire qui apparaît $(56,7 \%)$, alors que la mesure de la fluorescence reste encore élevée $(85,6 \%)$.

Après $24 \mathrm{~h}$ d'exposition de la suspension cellulaire aux facteurs stressants, la diminution du niveau de la fluorescence se poursuit mais reste à une valeur encore élevée (79\%). Par contre, les valeurs des activités respiratoire, métabolique et de l'intégrité membranaire sont faibles (respectivement 4, 2 et $11,8 \%$ ).

Au travers de cette cinétique, il apparaît que l'exposition aux trois facteurs stressants, entre autres le rayonnement UV, altère la physiologie des cellules de Salmonella Typhimurium C52 à des niveaux différents selon le temps d'exposition, dont la première conséquence est la perte très rapide du pouvoir de cultiver de ces cellules.

À partir de la cinétique des états physiologiques et dans le but d'évaluer le pouvoir pathogène des cellules non cultivables de Salmonella Typhimurium C52 vis-à-vis du modèle souris, trois temps d'exposition aux facteurs stressants ont été retenus afin d'obtenir des suspensions cellulaires VNC présentant des états physiologiques contrastés. Dans cette optique, quatre microcosmes sont respectivement soumis à $30 \mathrm{~min}, 1 \mathrm{~h}$ et $2 \times 24 \mathrm{~h}$ aux facteurs stressants. Les résultats des dénombrements cellulaires et des évaluations des états physiologiques obtenus dans chacun des microcosmes exposés sont présentés dans le tableau I et sur la figure 2. Dans cette expérience, l'activité métabolique, l'intégrité membranaire et la fluorescence des cellules stressées ont été prises en compte, l'activité respiratoire n'ayant pu être mesurée pour un problème de réactif.

Les résultals concernant les dénombrements des cellules cultivables et totales confirment ceux obtenus lors de l'étude de la cinétique (figure 1). Quelle que soil la durée
Tableau I. Valeurs des abondances des cellules cultivables el totales de Salmonella Typhimurium C52 dans quatre microcosmes exposés respectivement pendant $30 \mathrm{~min}, 1 \mathrm{~h}$ et $2 \times 24 \mathrm{~h}$ aux trois facteurs stressants.

Table I. Culurable and total cellular concentrations of Salmonello Typhimurium after $30 \mathrm{~min}$, one hour and $2 \times 24 \mathrm{~h}$ exposure time (four microcosms) to the three environmental factors.

\begin{tabular}{|c|c|c|c|c|}
\hline & $\begin{array}{c}\text { Microcosme } \\
\ll 30 \mathrm{~min} »\end{array}$ & $\begin{array}{l}\text { licrocosn } \\
\text { «1h } »\end{array}$ & $\begin{array}{c}\text { Licrocosn } \\
\text { I } \\
\ll 24 h »\end{array}$ & $\begin{array}{c}\text { Microcosmt } \\
\text { II } \\
« 24 \mathrm{~h} »\end{array}$ \\
\hline $\begin{array}{l}\text { Cellules } \\
\text { cultivables } / \mathrm{mL}\end{array}$ & 0,08 & 0.03 & 0 & 0 \\
\hline $\begin{array}{l}\log 10 \\
\text { (Cellules } \\
\text { totales }+1) / \mathrm{mL}\end{array}$ & 7,2 & 7.2 & 7,2 & 7.1 \\
\hline
\end{tabular}

de l'exposition aux facteurs stressants, les cellules de Salmonella Typhimurium C52 ne subissent aucune lyse, tandis que la perte du pouvoir de cultiver de ces cellules est presque totale dès $30 \mathrm{~min}$ d'exposition. Les mesures de l'activité métabolique des cellules des microcosmes « $30 \mathrm{~min} »$ et « $1 \mathrm{~h}$ » sont respectivement égales à 50 et $3 \%$ et vont dans le sens de ceux obtenus lors de l'étude de la cinétique. Les valeurs des mesures de l'intégrité membranaire sont également proches de celles obtenues lors de la cinétique $(>90 \%$ ), de même que pour les valeurs de la fluorescence. Les cellules exposées $24 \mathrm{~h}$ aux facteurs stressants, lors d'une expérience en double, présentent une activité métabolique nulle, une intégrité membranaire voisine de zéro et un niveau d'intensité de fluorescence de 60 et $75 \%$. Le bon niveau de concordance des résultats obtenus lors de ces deux expériences de $24 \mathrm{~h}$ d'exposition milite en faveur d'une constance dans les effets des facteurs stressants étudiés.

À partir de chacun des microcosmes exposés aux facteurs stressants, une suspension cellulaire de Salmonella Typhimurium C.52 est réalisée en vue de l'injection à la souris $(100 \mu \mathrm{L})$ sur laquelle sont contrôlés le niveau de la cultivabilité cellulaire et les différents états physiologiques des cellules bactériennes stressées (tableau $I I$ ).

Les trois centrifugations et lavages réalisés lors de la préparation des suspensions de cellules stressées constituent un facteur de stress supplémentaire intligé aux cellules ; les valeurs de l'intégrité membranaire et du niveau de la fluorescence sont inférieures de 10 à $20 \%$ par rapport à celles obtenues dans les microcosmes après exposition aux facteurs stressants. 


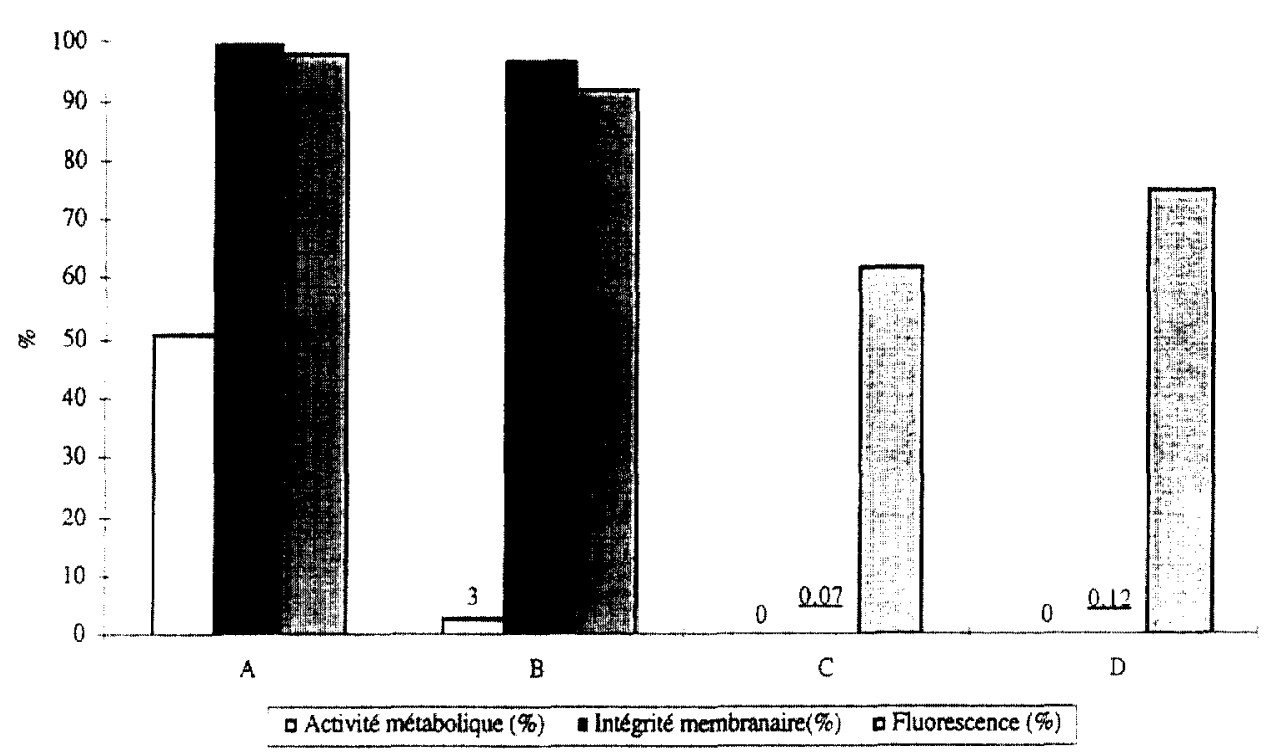

Figure 2. Évolution des pourcentages des différents états physiologiques que présentent les cellules de Salmonella Typhimurium en fonction du temps de l'exposition aux facteurs stressants. A (Microcosme $30 \mathrm{~min}$ ); B (Microcosme $1 \mathrm{~h}$ ); C (Microcosme I $24 \mathrm{~h}$ ); D (Microcosme II $24 \mathrm{~h}$ ).

Figure 2. Effects of simultaneous exposure to the three experimental factors over time on Salmonella Typhimurium cellular physiological states. A (Microcosm 30 min); B (Microcosm 1 h); C (Microcosm I 24 h); D (Microcosm Il 24 h).

Tableau II. Valeurs des abondances des cellules cultivables et totales et des pourcentages des états physiologiques des suspensions cellulaires stressées inoculées à la souris à partir des microcosmes exposés pendant des temps différents ( $30 \mathrm{~min}, 1 \mathrm{~h}$ et $2 \times 24 \mathrm{~h}$ ) aux facteurs stressants. Témoins pathogènes 1 et 2 : suspensions cellulaires non exposées aux facteurs stressants.

Table II. Salmonella Typhimurium cellular physiological states $(\%)$, number of culturable and total cells inoculated to mice after exposure to the three environmental factors ( $30 \mathrm{~mm}, \mathrm{I} \mathrm{h}, 24 \mathrm{~h} \mathrm{I}, 24 \mathrm{~h} / 1$ cellular suspensions). Pathogen controls 1 and 2: non-exposed cellular suspensions.

\begin{tabular}{|c|c|c|c|c|c|}
\hline & $\begin{array}{l}\text { Nb de cellules. } \\
\text { cultivables } \\
/ 100 \mu \mathrm{L}\end{array}$ & $\begin{array}{c}\text { Nb de cellules } \\
\text { totales } \\
/ 100 \mu \mathrm{L}\end{array}$ & $\begin{array}{c}\text { Activité } \\
\text { métabolique } \\
\%\end{array}$ & $\begin{array}{c}\text { Intégrité } \\
\text { membranaire } \\
\%\end{array}$ & $\begin{array}{c}\text { Fluorescence } \\
\%\end{array}$ \\
\hline Suspension cellulaire $30 \mathrm{~min}$ & 0 & $\begin{array}{c}9.6 .10^{2} \\
\pm 2.2\end{array}$ & 50 & 77.7 & 85,5 \\
\hline Suspension cellulaire $\mathrm{lh}$ & 0 & $\begin{array}{c}9,1.10^{2} \\
\pm 1,3\end{array}$ & $<6$ & 73 & 71 \\
\hline Suspension cellulaire $124 \mathrm{~h}$ & 0 & $\begin{array}{l}1,2.10^{3} \\
+(1.45\end{array}$ & 0 & 0 & 51.2 \\
\hline Suspension cellulaire II $24 \mathrm{~h}$ & 0 & $\begin{array}{l}6,6.10^{3} \\
\pm 0.6\end{array}$ & 0 & $<0.11$ & 71,4 \\
\hline $\begin{array}{l}\text { Témoin } \\
\text { pathogèrle } 1\end{array}$ & $4.10^{3}$ & $\begin{array}{c}4,1.10^{3} \\
\pm 0,6\end{array}$ & 82 & 58,9 & 100 \\
\hline $\begin{array}{l}\text { Témoin } \\
\text { pathogène } 2\end{array}$ & $4,5 \cdot 10^{3}$ & $\begin{array}{c}4.5 .10^{3} \\
\pm 1,1\end{array}$ & 77 & 78 & 100 \\
\hline
\end{tabular}

Lors de chaque injection de cellules de Salmonella Typhimurium C52 après exposition d'une durée déterminée aux effets des facteurs stressants à un lot de souris, des injections " témoin " sont réalisées en même temps sur d'autres lots de souris : une injection de cellules de Salmonella Typhimurium non exposées et donc non 
Tableau III. Effets de l'injection intra-péritonéale $(100 \mu \mathrm{L})$ de suspensions de cellules de Salmonella Typhimurium C52 soumises aux lacteurs stressants pendant des laps de temps différents $(30 \mathrm{~min} .1 \mathrm{~h}$ et $2 \times 24$ h) et de suspensions de cellules non soumises aux facteurs stressants (témoins pathogènes 1 et 2) à des lots de souris.

Table III. Effects of intraperitoneal injection of mice $(100 \mu \mathrm{L})$ with Salmonella Typhimurium C52 cellular suspensions exposed to the three experimental factors for variable times $(30 \mathrm{~min}, 1 \mathrm{~h}, 2 \times 24 \mathrm{~h}$ ) and non exposed cellular populations (pathogen controls).

\begin{tabular}{|c|c|c|c|c|c|c|c|}
\hline \multirow[t]{2}{*}{ Semaine } & \multirow{2}{*}{$\begin{array}{c}\text { Nb de } \\
\text { souris/lot }\end{array}$} & \multicolumn{4}{|c|}{ Mortalité cumulée par semaine } & \multirow[t]{2}{*}{ Mortalité totale } & \multirow{2}{*}{$\begin{array}{c}\text { Mortalité } \\
\%\end{array}$} \\
\hline & & lère & 2ème & 3ème & 4ème & & \\
\hline Suspension cellulaire $30 \mathrm{~min}$ & 10 & 0 & 0 & () & 0 & $0 / 10$ & 0 \\
\hline Suspension cellulaire $\mathrm{Ih}$ & 10 & 0 & 0 & 0 & 0 & $0 / 10$ & 0 \\
\hline Suspension cellulaire $124 \mathrm{~h}$ & 10 & 0 & 0 & 0 & 0 & $0 / 10$ & 0 \\
\hline Suspension cellulaire II $24 \mathrm{~h}$ & 10 & 0 & 0 & 0 & 0 & $0 / 10$ & 0 \\
\hline Témoin pathogène 1 & 10 & 10 & 0 & 0 & 0 & $10 / 10$ & 100 \\
\hline Témoin pathogène 2 & 10 & 10 & 0 & 0 & 0 & $10 / 10$ & 100 \\
\hline Témoin tampon Tris & 6 & 0 & 0 & 0 & 0 & $0 / 6$ & 0 \\
\hline
\end{tabular}

stressées (témoin pathogène) et une injection de Tampon Tris (pH 7,1) support de la suspension bactérienne (témoin négatif). L'injection intrapéritonéale aux souris de suspensions de cellules de Salmonella Typhimurium C52 (\# $1 \times 10^{3}$ cellules $100 \mu \mathrm{L}^{-1}$ ) après exposition aux facteurs stressants pendant $30 \mathrm{~min}, 1 \mathrm{~h}$ et $24 \mathrm{~h}$ n'entraîne aucune mortalité sur une durée de quatre semaines alors que la totalité de ces cellules a perdu son pouvoir de cultiver (tableau III). La mise en culture du foie et de la rate prélevés sur les souris inoculées avec les suspensions cellulaires exposées aux facteurs stressants témoigne de l'absence de cellules de Salmonella Typhimurium cultivables dans ces organes. Par contre, l'injection de cellules cultivables (\# $4 \times 10^{3}$ cellules $100 \mu \mathrm{L}^{-1}$ ), non stressées par le rayonnement UV, la salinité et l'oligotrophie, occasionne $100 \%$ de mortalité en une semaine dans les deux lots de souris (témoins pathogènes 1 et 2). Parallèlement, on constate la présence de cellules de Salmonella Typhimurium cultivables au niveau du foie et de la rate prélevés chez toutes ces souris inoculées.

Après 30 min d'exposition, la perte du pouvoir de cultiver s'accompagne d'une perte du pouvoir pathogène chez la souris et en même temps d'une diminution de l'activité métabolique cellulaire (50\%).

Après une exposition d'une heure aux facteurs stressants, les niveaux des états physiologiques sont peu différents de ceux des cellules exposées pendant $30 \mathrm{~min}$, à l'exception de l'activité métabolique qui est fortement atteinte $(<6 \%)$.

Après $24 \mathrm{~h}$ d'exposition des cellules aux facteurs stressants, les niveaux de leurs états physiologiques sont pratiquement nuls pour l'activité métabolique et l'intégrité membranaire et diminués de moitié dans la suspension cellulaire I et d'un tiers dans la suspension cellulaire Il pour le niveau de réémission de la fluorescence.

L'atteinte précoce de l'activité métabolique en même temps que la perte du pouvoir de cultiver pourrait faire penser que ces deux facteurs ont un rôle important dans la perte du pouvoir pathogène. Cependant, cette étude préliminaire ne permet pas, compte tenu du nombre d'essais, de confirmer ou d'infirmer que la perte du pouvoir pathogène est liée à la perte du pouvoir de cultiver et/ou au niveau de l'activité métabolique de la cellule bactérienne.

\section{DISCUSSION ET CONCLUSION}

L'exposition aux trois facteurs stressants des cellules de Salmonella Typhimurium induit systématiquement et rapidement un état cellulaire dit viable non cultivable, dont il est possible d'évaluer le niveau des atteintes des fonctions métaboliques en rapport avec la durée d'exposition à ces facteurs stressants.

Lors d'une étude sur l'inactivation de diverses bactéries pathogènes par le rayonnement UV, Chang et al. [6] mentionnent que pour Salmonella typhi une exposition de $2 \mathrm{~h}$ au rayonnement UV avec une intensité de 6 à 7 $\mathrm{mW} \mathrm{s} \mathrm{s}^{-1} \mathrm{~cm}^{-2}$ conduit à une réduction de 3 à $4 \log$ en cellules cultivables sans donner la valeur avant exposition. $\mathrm{La}$ conjonction de plusieurs facteurs stressants (rayonnement UV, salinité et oligotrophie) joue un rôle de synergie puisqu'avec une intensité de rayonnement UV comparable, les cellules de la souche Salmonella Typhimurium C52 présentent une perte du pouvoir de cultiver pratiquement 
totale dès 30 min d'exposition. Les cellules non cultivables conservent pendant les deux premières heures d'exposition aux trois facteurs stressants leur intégrité membranaire et un niveau de fluorescence voisin de ceux des cellules cultivables, tandis que leur activité métabolique (respiration et capacité de s'allonger en présence de nutriments) est fortement diminuée. Cette inhibition progressive du métabolisme cellulaire bactérien dans les deux premières heures d'exposition aux facteurs stressants pourrait ĉtre la conséquence d'une production accrue de photocomposés, par la composante UV des facteurs stressants, issus de la réduction de $\mathrm{l}^{\prime} \mathrm{O}_{2}$ (accepteur final d'électrons de la chaîne respiratoire) en produits hautement toxiques tels que $\mathrm{O}_{2}^{-}$, $\mathrm{H}_{2} \mathrm{O}_{2}, \mathrm{OH}$. À partir de la deuxième heure, l'impact des facteurs stressants se traduit par le début de la diminution du niveau de réémission de la fluorescence de l'ADN bactérien. L'atteinte de la structure de I'ADN cellulaire pourrait trouver son origine dans le processus de dimérisation entre les bases pyrimidiques adjacentes sur un même brin [25]. L'existence de liaisons covalentes se traduit par une accessibilité moindre des hases A-T pour le fluorochrome DAPI, d'où une diminution du niveau de la fluorescence réémise. Ces liaisons empêchent la réplication de l'ADN et conduisent à l'inactivation bactérienne. Une durée d'exposition plus longue, au-delà de $2 \mathrm{~h}$, est nécessaire pour observer une altération « physique » des cellules. Le pourcentage de l'altération de la membrane cytoplasmique des cellules soumises aux facteurs stressants pendant $24 \mathrm{~h}$ est proche de $100 \%$ alors que l'abondance des cellules totales reste constante, visualisant ainsi l'absence du phénomène de lyse cellulaire.

En ce qui concerne le facteur salinité, plusieurs auteurs considèrent que ce paramètre joue un rôle secondaire dans l'inactivation de Salmonella lors d'une courte période d'incubation en eau de mer $[9,28,29]$. Cependant, pour une incubation prolongée de Salmonella Typhimurium en microcosme eau de mer, Galès [14] montre que le rôle du facteur «salinité » est du même ordre d'importance que la partie UV du spectre solaire dans la disparition du nombre de cellules cultivables (CFU). En milieu marin, les entérobactéries, en l'occurrence Salmonella Typhimurium, peuvent dans certains cass, en fonction de leur vécu avant le rejet en mer [10], s'adapter au stress osmotique. Cependant, dans la présente étude, les conditions de stress auxquelles sont soumises les cellules de Salmonella Typhimurium C52, c'està-dire rayonncment UV, forte salinité et oligotrophie, sans préadaptation à une forte osmolarité et en l'absence d'osmoprotecteurs nécessaires à l'osmorégulation [23], entraînent une perte très rapide du pouvoir de cultiver des cellules bactériennes.

La persistance dans l'environnement de bactéries pathogènes viables non cultivables, et donc non détectées par les méthodes microbiologiques traditionnelles ( $\mathrm{CFU}$ ), pose le problème de la conservation ou non du pouvoir pathogène de ces cellules. Les résultats préliminaires obtenus dans cette étude montrent que la perte de cultivabilité de Salmonella Typhimurium C52, après une brève exposition aux trois facteurs stressants, entraîne lat perte du pouvoir pathogène de cette souche vis à vis de l'animal modèle, la souris, alors qu'une fraction élevée des cellules de la population bactérienne reste physiologiquement active. Bien qu'aucune étude n'ait été faite concernant Salmonella Typhimurium dans de telles conditions (application simultanée de trois facteurs stressants dont le rayonnement UV), il existe des travaux traitant de l'évolution de la virulence de plusieurs espèces bactériennes pathogènes viables non cultivables. Les conclusions des travaux des différents auteurs divergent selon le genre ou l'espèce bactérienne considéré, le facteur ayant induit l'état VNC et le mode d'évaluation du pouvoir pathogène (adhésion, invasion, production de toxines).

Pour Oliver et Bockian [32], les cellules de Vibrio vulnificus uniquement stressées par une incubation en eau de mer à $5^{\circ} \mathrm{C}$, présentent une baisse considérable de la virulence après injection intrapéritonéale chez la souris. Endommagées par le chlore, les cellules de Salmonella Typhimurium et d'E. coli, perdent leur capacité d'adhésion in vitro aux cellules épithéliales, et par conséquent perdent leur virulence avant de perdre leur cultivabilité [24]. Ce phénomène paraît lié à l'altération des pili et autres adhésines nécessaires à l'adhésion des bactéries [47]. Dans les mêmes conditions d'exposition au chlore, la perte de virulence des cellules de Yersinia VNC est causée non pas par la perte de la capacité d'adhésion mais par celle d'invasion [24].

Compte tenu de ces données bibliographiques, plusieurs hypothèses peuvent être émises quant à l'interprétation des résultats obtenus dans cette étude. Bien que les étapes d'adhésion et d'invasion des cellules bactériennes au niveau des cellules intestinales ne puissent être appréciées lors d'une injection intrapéritonéale, il est possible que certains déterminants de virulence de Salmonella Typhimurium nécessaires à la propagation de l'infection au-delà de la barrière intestinale aient été altérés.

La présence d'un plasmide $(90 \mathrm{~kb})$ est indispensable pour conférer aux cellules de Salmonella Typhimurium leur 
pleine virulence, c'est-à-dire pour que l'infection se propage au-delà de la barrière intestinale $[13,19,30,35]$. Ce plasmide intervient entre autres en favorisant la croissance de cette bactérie dans les tissus de l'hôte, en particulier ceux riches en macrophages (rate) $[13,20]$. Les effets des trois facteurs stressants, notamment le rayonnement UV, sur les cellules de Salmonella Typhimurium pourraient avoir des conséquences sur le maintien du plasmide de virulence de ces cellules et/ou sur son activité par d'éventuelles mutations. Certaines données bibliographiques concernant $E$. coli concluent au maintien des plasmides dans les cellules VNC lors de stress divers [5, 18]. Cependant, Palmer et al. [34] insistent sur la nécessité de recouvrement de la cultivabilité des cellules VNC, après incubation dans un milieu nutritif liquide (BHI, Difco) pour restituer une détection plasmidique positive.

Le rayonnement UV, connu pour ses propriétés mutagènes, pourrait occasionner en plus des troubles métaboliques cellulaires mis en évidence dans cette étude, des troubles aux conséquences structurales tels que la suppression de la production de cytotoxine, localisée au niveau de la membrane cytoplasmique ou encore l'altération de la structure du LPS (endotoxine impliquée dans la résistance à l'action lytique du complément) ou enfin des modifications se traduisant par l'atteinte de la phagocytose par les macrophages [11].

La perte de la virulence des cellules de Salmonella Typhimurium C52, observée dès 30 min d'exposition dans les conditions drastiques de l'expérimentation, pourrait être la conséquence de la perturbation du métabolisme général. De plus, pour une exposition prolongée au-delà d' $1 \mathrm{~h}$. il semblerait que des atteintes structurales de la membrane cytoplasmique (sans intervention du phénomène de lyse cellulaire) et de l'ADN (avec une perte de fluorescence) soient également impliquées.

L'examen du maintien et de l'activité du plasmide des cellules de Salmonella Typhimurium C52, devenues viables mais non cultivables après exposition aux trois facteurs stressants, fera l'objet d'une prochaine étude afin d'essayer de trouver une relation entre la perte ou l'inactivité du plasmide et la perte rapide du pouvoir pathogène de ces cellules de Salmonella Typhimurium.

\section{Remerciements}

Cette étude a été réalisée dans le cadre du Programme national d'océanographie côtière « microbiologie sanitaire » et a bénéficié du soutien financier afférent.

\section{RÉFÉRENCES}

[1] Allen-Austin D., Austin B., Colwell R.R., Survival of Aeromonas salmonicida in river water, FEMS Microbiol. Lett. 21 (1984) 143-146.

12] Baleux B., Got P., Apport de l'observation microscopique couplée à l'analyse d'images dans l'ćvaluation de la qualité bactériologique des eaux : approche cellulaire globale. T.S.M. 6 (1996) 430-436

[3] Barcina I., Gonzales J.M., Iriberri J., Egea L., Effect of visible light on progressive dormancy of Escherichia coli cells during the survival process in natural fresh water, Appl. Environ. Microbiol. 55 (1989) 246-251.

[4] Brauns L.A., Hudson M.C., Oliver J., Use of the polymerase chain reaction in detection of culturable and nonculturable Vibrio vulnificus cells, Appl. Environ. Microbiol. 57 (1991) $2651-2655$

[5] Byrd J.J., Colwell R.R., Maintenance of plasmids pBR322 and pUC8 in nonculturable Escherichia col $i$ in the marine environment, Appl. Environ. Microbjol. 56 (1990) 2104-2107.

[6] Chang J.C., Ossoff S.F., Lobe D.C., Dorfman M.H., Dumais C.M., Qualls R.G., Johnson J.D., UV inactivation of pathoge. nic and indicator microorganisms. Appl. Environ. Microbiol. 49 (1985) $1361-1365$.

17] Colwell R.R., Brayton P.R., Grimes D.J., Roszak D.B., Huq S.A., Palmer L.M., Viable but non-culturable Vibrio cholerae and related pathogens in the environment: implications for release of genetically engineered micronganisms, Bio/Technology 3 (1985) 817-820.

[8] Colwell R.R., Brayton P., Tamplin M., Huq S. Voll M., Roszak D., Muralidhar S.. Somerville C., Grimes I., Pathogenic potential including production of cholera toxin in viable but non recoverable Vibrio cholerae 01 in environmental microcosm, in: Kuwahara S., Pierce N.F., (Eds.) Advances in research on cholera and related diarrheas, 5th ed. KTK publishers, Tokyo, 1988.

[9] Cornax R., Morinigo M.A., Romero P., Borrego J.J., Survival of pathogenic microorganisms in seawater, Current Microbiol. 20)(1990) 293-298.

110] Csonka L.N., Hanson A.D. Prokaryotic osmoregulation: genetics and physiology, A. Rev. Microbiol. 45 (1991) 569 606 
[11] D'Aoust J-Y., Pathogenicity of foodborne Salmonella, International J. Food Microbiol. 12 (1991) 17-40.

[12] Desmonts C., Minet J., Colwell R., Cormier M., Fluorescentantibody method useful for detecting viable but nonculturable Salmonella spp. in chlorinated wastewater, Appl. Environ. Microbiol. 56 (1990) 1448-1452.

[13] Fierer J., Krause M., Tauxe R., Guiney D., Salmonella Typhimurium bacteremia: association with the virulence plasmid, $J$. Infectious Diseases 166 (1992) 639-642.

[14] Galès P., Origine et devenir d'une bactérie pathogène (Salmonella) dans les compartiments eau, sédiment, coquillages filtreurs d'un écosystème saumâtre côtier (étang de Thau), thèse doct. USTL II, Montpellier, France (1994) 218 p.

[15] Got P., Baleux B., Trousselier M., Dénombrements directs des bactéries des milieux aquatiques par microscopie en épifluorescence : comparaison entre un systeme d'analyse d'image automatisé (Mudicam) et l'observation visuelle, Rev. Sci. Eau 6 (1993) 269-284.

[16] Gourmelon M., Cillard J., Pommepuy M., Visible light damage to Escherichia coli in seawater: oxydative stress hypothesis, J. Appl. Bacteriol. 77 (1994) 105-112.

[17] Grimes D.J., Atwell R.W., Brayton P.R., Palmer L.M., Rollins D.M., Roszak D.B., Singleton F.L., Tamplin M.L., Colwell R.R., The fate of enteric pathogenic bacteria in estuarine and marine environments, Microbiol. Sciences 3 (1986) 324-329.

[18] Grimes D.J., Colwell R.R., Viability and virulence of Escherichia coli suspended by membrane chamber in semi-tropical ocean water, FEMS Microbiol. Lett. 34 (1986) 161-165.

[19] Gulig P., Virulence plasmids of Salmonella Typhimurium and other salmonellae, Mini review, Microb. Pathog. 8 (1990) 3-11.

[20] Gulig P.A., Doyle T.J., The Salmonella Typhimurium virulence plasmid increases the growth rate of Salmonella in mice, Infect. Immun. 61 (1993) 504-511.

[21] Jones D.M., Sutcliffe E.M., Curry A., Recovery of viable but non culturable Campylobacter jejuni, J. Gen. Microbiol. 25 (1991) 415-420.

[22] Kogure K., Simidu U., 'Taga N., A tentative direct microscopy method for counting living marine bacteria, Can. J. Microbiol. 25 (1979) 415-420.

[23] Le Rudulier D., L'osmorégulation chez les bactéries : aspects physiologiques et génétiques, Bull. Soc. Fr. Microbiol. 8 (1993) $167-169$.

[24] LeChevallier M.W., Singh A., Schiemann D.A., McFetcrs G.A., Changes in virulence of waterbome enteropathogens with chlorine injury, Appl. Environ. Microbiol. 50 (1985) 412-419.

[25] Lindenauer K.G., Darby J.L., Ultraviolet disinfection of wastewater: effect of dose on subsequent photoreactivation, Wat. Res. 28 (1994) 805-817.

[26] Linder K., Oliver J., Membrane fatty acid and virulence changes in the viable but non culturable state of Vibrio valnificus, Appl. Environ. Microbiol. 55 (1989) 2837-2842.
[27] McKay A.M., Viable but non-culturable forms of potentially pathogenic bacteria in water, Lett. Appl. Bacteriol. 14 (1992) 129-135.

[28] Morinigo M.A., Cornax R., Castro D., Martinez-Manzanares E., Borrego J.J., Viability of Salmonella spp and indicator microorganisms in seawater using membrane diffusion chambers, Antonie van Leeuwenhoek 57 (1990) 109-117.

[29] Morinigo M.A., Cornax R., Munoz M.A., Romero P., Borrego J.J., Viability of Salmonella species in natural waters, Current Microbiol.18 (1989) 267-273.

[30] Norel F., Coynault C., Miras I., Hermant D., Popoff M.Y., Cloning and expression of plasmid DNA sequences involved in Salmonella serotype Typhimurium virulence, Mol. Microbiol. 3 (1989) 733-743.

[31] Oliver J.D., Nonculturability and resuscitation of Vibrio vulnificus, Trends in Microbiol Ecology, Guerrero R., Pedros-Alio C. (Eds.), 1993, pp. 187-191.

[32] Oliver J.D., Bockian R., In vivo rescucitation, and virulence towards mice, of viable but non culturable cells of Vibrio vulnificus, Appl. Environ. Microbiol. 61 (1995) 2620-2623.

[33] Oliver J.D., Hite F., McDougald D., Andon L.N., Simpsun L.M., Entry into, and resuscitation from, the viable but non culturable state by Vibrio vulnificus in an estuarine environment, Appl. Environ. Microbiol. 61 (1995) 2624-2630.

[34] Palmer L.M., Baya A.M., Grimes D.J., Colwell R.R., Molecular genetic and phenotypic alteration of Escherichia coli in natural water microcosms containing toxic chemicals, FEMS Microbiol. Letters 21 (1984) 169-173.

[35] Pardon P., Popoff M.Y., Coynault C., Marly J., Miras I., Virulence-associated plasmids of Salmonella serotype Typhimurium in experimental murine infection, Ann. Inst. Pasteur/ Microbiol. 137 B (1986) 47-60.

[36] Porter K.G., Feig Y.S., The use of DAPI for identifying and counting aquatic microflore, Limnol. Oceanogr. 25 (1980) 943-949.

[37) Rahman I., Shahamat M., Chowdhury M.A.R., Colwell R.R., Potential virulence of viable but non culturable Shigella dvsenteria type 1. Appl. Environ. Microbiol. 62 (1996) 115-120.

[38] Rahman I., Shahamat M., Kirchman P.A., Russek-Cohen E. Colwell R.R.. Methionine uptake and cytopathogenicity of viable but nonculturable Shigella dysenterio Type 1. Appl. Environ. Microbiol. 60 (1994) 3573-3578.

[39] Rodriguez G.G., Phipps D., Ishiguro K.. Ridgway use of a fluorescent redox probe for direct visualization of actively respiring bacteria, Appl. Environ. Microbiol. 58 (1992) 18011808

[40] Rollins D.M., Colwell R.R., Viable but non culturable stage of Campylobacter jejuni and its role in survival in the natural aquatic environment, Appl. Environ. Microbiol. 52 (1986) $531-538$

[41] Roszak D.B., Colwell R.R., Survival strategies of bacteria in the natural environment, Microb. Reviews 51 (1987) 365-379.

[42] Roszak D.B., Colwell R.R., Metabolic activity of bacterial cells enumerated by direct viable count. Appl. Environ. Microbiol., 53 (1987) 2889-2983. 
[43] Roszak D.B., Grimes D.J., Colwell R.R., Viable but nonculturable stage of Salmonella enteritidis in aquatic systems, Can. J. Microbiol. 30 (1984) 334-338.

[44] Singh A., LeChevallier M.W., McFeters G.A., Reduced virulence of Yersinia enterocolitica by copper-induced injury, Appl. Environ. Microbiol. 50 (1985) 406-411.

[45] Singh A., Yeager R., McFeters G.A., Assessment of in vivo revival, growth, and pathogenicity of Escherichia coli strains after copper- and chlorinc-induced injury, Appl. Environ Microbiol. 52 (1986) 832-837.
[46] Singh A., Mc Feters G.A., Recovery, growth and production of heat-stable enterotoxin by Escherichia coli after copper-induced injury, Appl. Environ. Microbiol. 51 (1986) 738-742.

[47] Walsh S.M., Bissonnette G.K., Chlorine-induced damage to surface adhesins during sublethal injury of enterotoxigenic Escherichia coli, Appl. Environ. Microbiol. 45 (1983) 10601065.

[48] Xu H-S, Roberts N., Singleton F.L., Attwell R.W., Grimes D.J., Colwell R.R., Survival and viability of nonculturable Escherichia coli and Vibrio cholerae in the estuarinc and marine environment, Microb. Ecol. 8 (1982) 313-323. 